Supporting Information to

\title{
Dissection of Interaction Kinetics through Single-molecule Interaction Simulation
}

\author{
Manhua Pan, Yuteng Zhang, Guangjie Yan, Tai-Yen Chen* \\ Department of Chemistry, University of Houston, Houston, Texas 77204, United States. \\ ${ }^{*}$ Correspondence: tchen37@ central.uh.edu
}

\section{Table of content}

1. Derivation of analytical expression of $f(\tau),\langle\tau\rangle^{-1}$ of two FRET states model 2

1.1 Analytical expression of $f_{\mathrm{HL}}(\tau),\left\langle\tau_{\mathrm{HL}}\right\rangle^{-1}, P_{\mathrm{ES}}(t), P_{\mathrm{E}}(t), P_{\mathrm{ES} *}(t)$ of $\operatorname{high}\left(I_{\mathrm{H}}\right)$ to $\operatorname{low}\left(I_{\mathrm{L}}\right)$

1.2 Analytical expression of $f_{\mathrm{LH}}(\tau),\left\langle\tau_{\mathrm{LH}}\right\rangle^{-1}, P_{\mathrm{ES}}(t), P_{\mathrm{E}}(t), P_{\mathrm{ES} *}(t)$ of $\operatorname{low}\left(I_{\mathrm{L}}\right)$ to high $\left(I_{\mathrm{H}}\right)$

1.3 Analytical expression of $P_{\mathrm{ES}}([\mathrm{S}]), P_{\mathrm{E}}([\mathrm{S}]), P_{\mathrm{ES}} *([\mathrm{~S}])$ of two-state model 5

2. Computation cost for SMIS simulation and fittings $\quad 6$

3. SMIS validation for Michaelis-Menten model (other four conditions) 6

$3.1 \quad$ Validation of simulated $f_{\mathrm{HL}}(\tau)$ and $f_{\mathrm{LH}}(\tau)$ as a function of $k \quad 7$

3.2 Validation of simulated $\left\langle\tau_{\mathrm{HL}}\right\rangle^{-1}$ and $\left\langle\tau_{\mathrm{LH}}\right\rangle^{-1}$ as a function of $k$

3.3 Validation of of simulated $P_{\mathrm{E}}([\mathrm{S}]), P_{\mathrm{ES} *([\mathrm{~S}]) \text {, and } P_{\mathrm{ES}}([\mathrm{S}]) \text { as a function of } k} k$

4. SMIS validation using a kinetic model having five reaction species in 3 FRET levels $\quad 8$ 


\section{Derivation of analytical expression of $f(\tau),\langle\tau\rangle^{-1}$ of two FRET states model}

In this section, we derived the analytical expression of the probability density function of dwell time, $f(\tau)$, of the two-state model presented in Figure 1. First, we divided the kinetic model into high to low $\left(I_{\mathrm{H}}\right.$ to $I_{\mathrm{L}}$, Figure S1) and low to high $\left(I_{\mathrm{L}}\right.$ to $I_{\mathrm{H}}$, Figure S2) to solved for the $f_{\mathrm{HL}}(\tau)$ and $f_{\mathrm{LH}}(\tau)$, respectively. In each sub-model, the analytical expression of probability density functions $f(\tau)$, average transition rate $\langle\tau\rangle^{-1}$ (Section 1.1 and 1.2), and their relative populations $P_{i}([\mathrm{~S}])$ (Section 1.3) were solved by the singlemolecule rate equations.

\subsection{Analytical expression of $f_{\mathrm{HL}}(\tau),\left\langle\tau_{\mathrm{HL}}\right\rangle^{-1}, P_{\mathrm{ES}}(t), P_{\mathrm{E}}(t), P_{\mathrm{ES}}(t)$ of high $\left(I_{\mathrm{H}}\right)$ to $\operatorname{low}\left(I_{\mathrm{L}}\right)$ model}

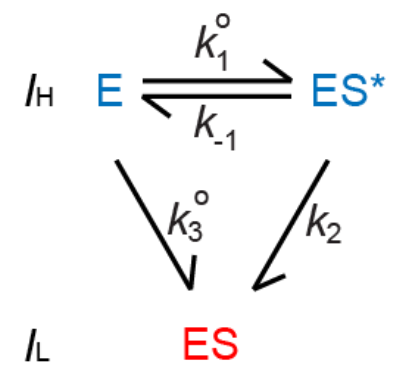

Figure S1. Proposed $\boldsymbol{I}_{\mathrm{H}}$ to $\boldsymbol{I}_{\mathrm{L}}$ model. E: enzyme; ES*: intermediate; ES: interacting complex.

To derive the $f_{\mathrm{HL}}(\tau)$ in the two-state kinetic model, we focused on the steps involve the transition from $\mathrm{E} \rightarrow \mathrm{ES}$ and $\mathrm{ES}^{*} \rightarrow \mathrm{ES}$ (Figure S1). The single-molecule rate equations for these steps are

$$
\begin{gathered}
\frac{d P_{\mathrm{ES}}(t)}{d t}=k_{3}{ }^{\mathrm{o}} P_{\mathrm{E}}(t)+k_{2} P_{\mathrm{ES}^{*}}(t) \\
\frac{d P_{\mathrm{E}}(t)}{d t}=k_{-1} P_{\mathrm{ES}^{*}}(t)-k_{1}{ }^{\mathrm{o}} P_{\mathrm{E}}(t)-k_{3}{ }^{\mathrm{o}} P_{\mathrm{E}}(t) \\
\frac{d P_{\mathrm{ES}^{*}}(t)}{d t}=k_{1}{ }^{\mathrm{o}} P_{\mathrm{E}}(t)-k_{-1} P_{\mathrm{ES}^{*}}(t)-k_{2} P_{\mathrm{ES}^{*}}(t)
\end{gathered}
$$

, where the $P_{i}(\mathrm{t})$ 's are the probabilities of finding the species $(i=\mathrm{E}, \mathrm{ES} *$, and ES) in the corresponding states at time $t$. Note that ${k_{1}}^{\mathrm{o}}=k_{1}[\mathrm{~S}]$ and ${k_{3}}^{\mathrm{o}}=k_{3}[\mathrm{~S}]$. Both ${k_{1}}^{\mathrm{o}}$ and ${k_{3}}^{\mathrm{o}}$ are treated as pseudo-first-order rate constants.

Transitioning from the $I_{\mathrm{L}}$ to $I_{\mathrm{H}}$ state can occur either through the ES $\rightarrow$ E or ES $\rightarrow$ ES* pathways, which gives two initial conditions: (1) initial species is $\mathrm{E}: P_{\mathrm{E}}(0)=1, P_{\mathrm{ES} *}(0)=0, P_{\mathrm{ES}}(0)=0$; and (2) initial species is $\mathrm{ES}^{*}: P_{\mathrm{E}}(0)=0, P_{\mathrm{ES} *}(0)=1, P_{\mathrm{ES}}(0)=0$. The probability function of time $t, P_{\mathrm{ES}}(t), P_{\mathrm{E}}(t), P_{\mathrm{ES} *}(t)$, can be obtained by solving the Eq S1 to S3 with initial conditions:

Inital condition 1: (1) $\mathrm{t}=0, P_{\mathrm{E}}(0)_{1}=1, P_{\mathrm{ES} *(0)}\left(\mathbf{1}=0, P_{\mathrm{ES}}(0)_{1}=0\right.$.

$$
P_{\mathrm{E}}(t)_{1}=I_{1} \mathrm{e}^{(\mathrm{B}+\mathrm{A}) t}+I_{2} \mathrm{e}^{(\mathrm{B}-\mathrm{A}) t}
$$

, where

$$
\begin{aligned}
& \mathrm{A}=\frac{\sqrt{\left(k_{-1}+{k_{3}}^{\mathrm{o}}+k_{2}+{k_{1}}^{\mathrm{o}}\right)^{2}-4\left({k_{3}}^{\mathrm{o}} k_{-1}+k_{2}\left({k_{1}}^{\mathrm{o}+k_{3}}{ }^{\mathrm{o}}\right)\right)}}{2}, \mathrm{~B}=\frac{-\left(k_{3}{ }^{\mathrm{o}}+k_{2}+k_{1}{ }^{\mathrm{o}}+k_{-1}\right)}{2}, \\
& I_{1}=\frac{1}{2 A}\left(\mathrm{~A}+\mathrm{B}+k_{2}+k_{-1}\right), I_{2}=\frac{1}{2 A}\left(\mathrm{~A}-\mathrm{B}-k_{2}-k_{-1}\right),
\end{aligned}
$$




$$
\begin{aligned}
& P_{\mathrm{ES}^{*}}(t)_{1}=\frac{k_{1}{ }^{\mathrm{o}}}{2 A}\left[\mathrm{e}^{(\mathrm{B}+\mathrm{A}) t}-\mathrm{e}^{(\mathrm{B}-\mathrm{A}) t}\right] \\
& P_{\mathrm{ES}}(t)_{1}=1+\frac{1}{2 A}\left[\left(k_{3}^{\mathrm{o}}+\mathrm{B}-\mathrm{A}\right) \mathrm{e}^{(\mathrm{B}+\mathrm{A}) t}-\left({k_{3}}^{\mathrm{o}}+\mathrm{B}+\mathrm{A}\right) \mathrm{e}^{(\mathrm{B}-\mathrm{A}) t}\right]
\end{aligned}
$$

Initial condition 2: (2) $\mathrm{t}=0, P_{\mathrm{E}}(0)_{2}=0, P_{\mathrm{ES} *(0)_{2}}=1, P_{\mathrm{ES}}(0)_{2}=0$.

$$
\begin{gathered}
P_{\mathrm{E}}(t)_{2}=\frac{k_{-1}}{2 A}\left[\mathrm{e}^{(\mathrm{B}+\mathrm{A}) t}-\mathrm{e}^{(\mathrm{B}-\mathrm{A}) t}\right] \\
P_{\mathrm{ES}^{*}(t)_{2}}=I_{2} \mathrm{e}^{(\mathrm{B}+\mathrm{A}) t}+I_{1} \mathrm{e}^{(\mathrm{B}-\mathrm{A}) t} \\
P_{\mathrm{ES}}(t)_{2}=1+\frac{1}{2 A}\left[\left(k_{2}+\mathrm{B}-\mathrm{A}\right) \mathrm{e}^{(\mathrm{B}+\mathrm{A}) t}-\left(k_{2}+\mathrm{B}+\mathrm{A}\right) \mathrm{e}^{(\mathrm{B}-\mathrm{A}) t}\right]
\end{gathered}
$$

The total probability function of each interacting species equals to:

$$
P_{i}(t)=C_{1} P_{i}(t)_{1}+C_{2} P_{i}(t)_{2}(i=\mathrm{E}, \mathrm{ES} * \text { or } \mathrm{ES})
$$

, where $C_{1}$ and $C_{2}$ are the probability coefficients for two different initial conditions. The $P_{i}(\mathrm{t})$ can be further simplified and summarized in Eq S11 to S13.

$$
P_{\mathrm{E}}(t)=J_{1} \mathrm{e}^{(\mathrm{B}+\mathrm{A}) t}+J_{2} \mathrm{e}^{(\mathrm{B}-\mathrm{A}) t}
$$

, where

$$
J_{1}=\frac{k_{-1} k_{-2}+2 A I_{1} k_{-3}}{2 A\left(k_{-2}+k_{-3}\right)}, J_{2}=-\frac{k_{-1} k_{-2}+2 A I_{2} k_{-3}}{2 A\left(k_{-2}+k_{-3}\right)},
$$

$$
P_{\mathrm{ES}^{*}}(t)=M_{1} \mathrm{e}^{(\mathrm{B}+\mathrm{A}) t}+M_{2} \mathrm{e}^{(\mathrm{B}-\mathrm{A}) t}
$$

, where

$$
\begin{aligned}
M_{1}=\frac{k_{1}{ }^{0} k_{-3}+2 \mathrm{~A} I_{2} k_{-2}}{2 A\left(k_{-2}+k_{-3}\right)}, M_{2}= & -\frac{k_{1}{ }^{0} k_{-3}+2 \mathrm{~A} I_{1} k_{-2}}{2 A\left(k_{-2}+k_{-3}\right)} \\
& P_{\text {ES }}(t)=1+N_{1} \mathrm{e}^{(\mathrm{B}+\mathrm{A}) t}-N_{2} \mathrm{e}^{(\mathrm{B}-\mathrm{A}) t}
\end{aligned}
$$

, where

$$
N_{1}=\frac{\left(\mathrm{B}-\mathrm{A}+k_{2}\right) k_{-2}+\left(\mathrm{B}-\mathrm{A}+k_{3}\right) k_{-3}}{2 A\left(k_{-2}+k_{-3}\right)}, N_{2}=\frac{\left(\mathrm{A}+\mathrm{B}+k_{2}\right) k_{-2}+\left(\mathrm{A}+\mathrm{B}+k_{3}\right) k_{-3}}{2 A\left(k_{-2}+k_{-3}\right)} .
$$

$\tau_{\mathrm{HL}}$ is the time needed to complete steps involving $k_{1}{ }^{\circ}, k_{3}{ }^{\circ}, k_{-1}$, and $k_{2}$. The probability of finding a particular $\tau$ is equal to the probability for the enzyme switch from E or ES* to the ES state between $t=\tau$ and $\tau+\Delta \tau$. Mathematically, this probability is equal to $\Delta P_{\mathrm{ES}}(\tau)$. In the limit of infinitesimal $\Delta \tau$, the probability density function of dwell time $\tau_{\mathrm{HL}}, f_{\mathrm{HL}}(\tau)$, can be obtained from $f_{\mathrm{HL}}(\tau)=\frac{d P_{\mathrm{ES}}(\tau)}{d \tau}$. Because of the presence of two ES formation pathways, we can first derive the $f_{\mathrm{HL}}(\tau)_{\mathrm{i}}$ for each pathway.

$$
f_{\mathrm{HL}}(\tau)_{1}=\frac{d P_{\mathrm{ES}}(\tau)_{1}}{d \tau}=Q_{1} \mathrm{e}^{(\mathrm{B}+\mathrm{A}) \tau}+Q_{2} \mathrm{e}^{(\mathrm{B}-\mathrm{A}) \tau}
$$

, where $Q_{1}=\frac{k_{3}{ }^{\mathrm{o}}\left(\mathrm{A}+\mathrm{B}+k_{2}+k_{-1}\right)+k_{1}{ }^{\mathrm{o}} k_{2}}{2 \mathrm{~A}}, Q_{2}=\frac{{k_{3}}^{\mathrm{o}}\left(\mathrm{A}+\mathrm{B}+k_{1}{ }^{\mathrm{o}}+k_{3}{ }^{\mathrm{o}}\right)-k_{1}{ }^{\mathrm{o}} k_{2}}{2 \mathrm{~A}}$

$$
f_{\mathrm{HL}}(\tau)_{2}=\frac{d P_{\mathrm{ES}}(\tau)_{2}}{d \tau}=R_{1} \mathrm{e}^{(\mathrm{B}+\mathrm{A}) \tau}+R_{2} \mathrm{e}^{(\mathrm{B}-\mathrm{A}) \tau}
$$

, where $R_{1}=\frac{k_{2}\left(\mathrm{~A}+\mathrm{B}+k_{1}{ }^{0}+k_{3}{ }^{o}\right)+k_{3}{ }^{o} k_{-1}}{2 \mathrm{~A}}, R_{2}=\frac{k_{2}\left(\mathrm{~A}+\mathrm{B}+k_{2}+k_{-1}\right)-k_{3}{ }^{\mathrm{o}} k_{-1}}{2 \mathrm{~A}}$ 
The analytical expression of overall probability density function $f_{\mathrm{HL}}(\tau)$ can be obtained as follows:

$$
f_{\mathrm{HL}}(\tau)=C_{1} f_{\mathrm{HL}}(\tau)_{1}+C_{2} f_{\mathrm{HL}}(\tau)_{2}
$$

Here, $C_{1}$ and $C_{2}$ are the coefficients representing the likelihoods of the initial species of $\mathrm{E}$ and $\mathrm{ES} *$, respectively. Since $C_{1}$ and $C_{2}$ include all possible ways to generate $\mathrm{E}$ and ES*, we can write $C_{1}+C_{2}=1$. The ratio of $C_{1}$ to $C_{2}$ is equal to the ratio of respective rate constant through path involved $k_{-3}$ to path involved $k_{-2}$ as shown in Figure $\mathbf{S 2}$ (i.e., $\frac{C_{1}}{C_{2}}=\frac{k_{-3}}{k_{-2}}$ ). Therefore, we got $C_{1}=\frac{k_{-3}}{k_{-3}+k_{-2}}, C_{2}=\frac{k_{-2}}{k_{-3}+k_{-2}}$, and Eq S17.

$$
f_{\mathrm{HL}}(\tau)=\frac{k_{-3}}{k_{-2}+k_{-3}} f_{\mathrm{HL}}(\tau)_{1}+\frac{k_{-2}}{k_{-2}+k_{-3}} f_{\mathrm{HL}}(\tau)_{2}
$$

The $f_{\mathrm{HL}}(\tau)$ was further simplified as

$$
f_{\mathrm{HL}}(\tau)=D_{1} \mathrm{e}^{(\mathrm{B}+\mathrm{A}) t}+D_{2} \mathrm{e}^{(\mathrm{B}-\mathrm{A}) t}
$$

, where $D_{1}=\frac{\left(k_{2}\left(\mathrm{~A}+\mathrm{B}+{k_{1}}^{\mathrm{o}}+{k_{3}}^{\mathrm{o}}\right)+{k_{3}}^{\mathrm{o}} k_{-1}\right) k_{-2}+\left(k_{1}{ }^{\mathrm{o}} k_{2}+k_{3}{ }^{\mathrm{o}}\left(\mathrm{A}+\mathrm{B}+k_{2}+k_{-1}\right)\right) k_{-3}}{2 \mathrm{~A}\left(k_{-2}+k_{-3}\right)}$,

$$
D_{2}=\frac{\left(k_{2}\left(\mathrm{~A}+\mathrm{B}+k_{2}+k_{-1}\right)-k_{3}{ }^{\mathrm{o}} k_{-1}\right) k_{-2}+\left(-k_{1}{ }^{\mathrm{o}} k_{2}+k_{3}{ }^{\mathrm{o}}\left(\mathrm{A}+\mathrm{B}+k_{1}{ }^{\mathrm{o}}+k_{3}{ }^{\mathrm{o}}\right)\right) k_{-3}}{2 \mathrm{~A}\left(k_{-2}+k_{-3}\right)}
$$

With $f_{\mathrm{HL}}(\tau)$, we calculated the average transition time $\left\langle\tau_{\mathrm{HL}}\right\rangle$ through $\left\langle\tau_{\mathrm{HL}}\right\rangle=\int_{0}^{\infty} \tau f_{\mathrm{HL}}(\tau) d \tau$. The reciprocal of $\left\langle\tau_{\mathrm{HL}}\right\rangle$ gave the average transition rate, $\left\langle\tau_{\mathrm{HL}}\right\rangle^{-1}$, which is expressed as Eq S19:

$$
\begin{aligned}
\left\langle\tau_{\mathrm{HL}}\right\rangle^{-1}=\left(\frac{k_{-3}\left(k_{2}+k_{-1}+k_{1}[\mathrm{~S}]\right)+k_{-2}\left(k_{-1}+\left(k_{1}+k_{3}\right)[\mathrm{S}]\right)}{\left(k_{-2}+k_{-3}\right)\left(k_{1} k_{2}+k_{3}\left(k_{2}+k_{-1}\right)\right)[\mathrm{S}]}\right)^{-1} & \left(k_{-2}+k_{-3}\right)\left(k_{1} k_{2}+k_{3}\left(k_{2}+k_{-1}\right)\right)[\mathrm{S}] \\
= & \frac{\left(k_{-3}\left(k_{2}+k_{-1}+k_{1}[\mathrm{~S}]\right)+k_{-2}\left(k_{-1}+\left(k_{1}+k_{3}\right)[\mathrm{S}]\right)\right.}{k_{-1}}
\end{aligned}
$$

\subsection{Analytical expression of $f_{\mathrm{LH}}(\tau),\left\langle\tau_{\mathrm{LH}}\right\rangle^{-1}, P_{\mathrm{ES}}(t), P_{\mathrm{E}}(t), P_{\mathrm{ES}}(t)$ of $\operatorname{low}\left(I_{\mathrm{L}}\right)$ to high $\left(I_{\mathrm{H}}\right) \operatorname{model}$}

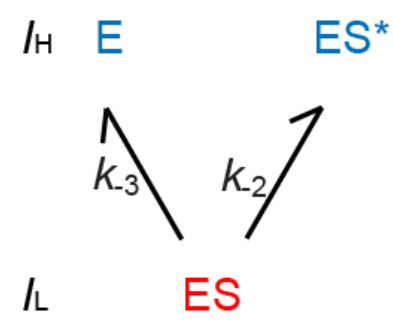

Figure S2. Proposed $\boldsymbol{I}_{\mathrm{L}}$ to $\boldsymbol{I}_{\mathbf{H}}$ model. E: enzyme; ES*: intermediate; ES: interacting complex.

To derive the $f_{\mathrm{LH}}(\tau)$ in the two-state kinetic model, we focused on the steps involve the transition from $\mathrm{ES} \rightarrow \mathrm{E}$ and $\mathrm{ES} \rightarrow \mathrm{ES}^{*}$ (Figure S2). The single-molecule rate equations for these steps are

$$
\frac{d P_{\mathrm{ES}}(t)}{d t}=-k_{-3} P_{\mathrm{ES}}(t)-k_{-2} P_{\mathrm{ES}}(t)
$$




$$
\begin{gathered}
\frac{d P_{\mathrm{E}}(t)}{d t}=k_{-3} P_{\mathrm{ES}}(t) \\
\frac{d P_{\mathrm{ES}^{*}}(t)}{d t}=k_{-2} P_{\mathrm{ES}}(t)
\end{gathered}
$$

Here, the initial condition is: $\mathrm{t}=0, P_{\mathrm{E}}(0)=0, P_{\mathrm{ES} *}(0)=0, P_{\mathrm{ES}}(0)=1$. Thus, the probability function of time $t, P_{\mathrm{ES}}(t), P_{\mathrm{E}}(t), P_{\mathrm{ES}}(t)$, was obtained by solving the above Eq S20 to S22 with the initial condition:

$$
\begin{array}{r}
P_{\mathrm{E}}(t)=\frac{k_{-3}\left(1-\mathrm{e}^{-\left(k_{-2}+k_{-3}\right) t}\right)}{k_{-2}+k_{-3}} \\
P_{\mathrm{ES}}(t)=\mathrm{e}^{-\left(k_{-2}+k_{-3}\right) t} \\
P_{\mathrm{ES}^{*}}(t)=\frac{k_{-2}\left(1-\mathrm{e}^{-\left(k_{-2}+k_{-3}\right) t}\right)}{k_{-2}+k_{-3}}
\end{array}
$$

$\tau_{\mathrm{LH}}$ is the time needed to complete steps involving $k_{-2}$, and $k_{-3}$. The probability of finding a particular $\tau$ is equal to the probability for the enzyme switch from ES to the E or ES* state between $t=\tau$ and $\tau+$ $\Delta \tau$. Mathematically, this probability is equal to $\Delta P_{\mathrm{E}}(\tau)+\Delta P_{\mathrm{ES} *}(\tau)$. In the limit of infinitesimal $\Delta \tau$, the probability density function of dwell time $\tau_{\mathrm{HL}}, f_{\mathrm{HL}}(\tau)$, was obtained from $f_{\mathrm{HL}}(\tau)=\frac{d P_{\mathrm{E}}(\tau)}{d \tau}+\frac{d P_{\mathrm{ES}}(\tau)}{d \tau}$.

$$
f_{\mathrm{LH}}(\tau)=\frac{d P_{\mathrm{E}}(\tau)}{d \tau}+\frac{d P_{\mathrm{ES}}(\tau)}{d \tau}=\left(k_{-2}+k_{-3}\right) \mathrm{e}^{-\left(k_{-2}+k_{-3}\right) \tau}
$$

With $f_{\mathrm{LH}}(\tau)$, we calculated the average transition time $\left\langle\tau_{\mathrm{LH}}\right\rangle$ through $\left\langle\tau_{\mathrm{LH}}\right\rangle=\int_{0}^{\infty} \tau f_{\mathrm{LH}}(\tau) d \tau$. The reciprocal of $\left\langle\tau_{\mathrm{LH}}\right\rangle$ gave the average transition rate, $\left\langle\tau_{\mathrm{LH}}\right\rangle^{-1}$, which is expressed as Eq S27:

$$
\left\langle\tau_{\mathrm{LH}}\right\rangle^{-1}=k_{-2}+k_{-3}
$$

\subsection{Analytical expression of $P_{\mathrm{ES}}([\mathrm{S}]), P_{\mathrm{E}}([\mathrm{S}]), P_{\mathrm{ES}}([\mathrm{S}])$ of two-state model}

Subploplution of each interaction species (i.e., $P_{\mathrm{E}}, P_{\mathrm{ES}}, P_{\mathrm{ES}}$ ) is a function of substrate concentration and time, denoted as $P_{\mathrm{E}}([\mathrm{S}], t), P_{\mathrm{ES}}([\mathrm{S}], t), P_{\mathrm{ES}}([\mathrm{S}], t)$. The single-molecule rate equations for the twostate kinetic model are:

$$
\begin{aligned}
& \frac{d P_{\mathrm{E}}([\mathrm{S}], t)}{d t}=k_{-1} P_{\mathrm{ES}}^{*}([\mathrm{~S}], t)+k_{-3} P_{\mathrm{ES}}([\mathrm{S}], t) \\
& -\left(k_{1}+k_{3}\right)[\mathrm{S}] P_{\mathrm{E}}([\mathrm{S}], t) \\
& \frac{d P_{\mathrm{ES}^{*}}([\mathrm{~S}], t)}{d t}=k_{1}[\mathrm{~S}] P_{\mathrm{E}}([\mathrm{S}], t)+k_{-2} P_{\mathrm{ES}}([\mathrm{S}], t)-\left(k_{-1}+k_{2}\right) P_{\mathrm{ES}}^{*}([\mathrm{~S}], t) \\
& \frac{d P_{\mathrm{ES}}([\mathrm{S}], t)}{d t}=k_{3}[\mathrm{~S}] P_{\mathrm{E}}([\mathrm{S}], t)+k_{2} P_{\mathrm{ES}}{ }^{*}([\mathrm{~S}], t)-\left(k_{-3}+k_{-2}\right) P_{\mathrm{ES}}([\mathrm{S}], t)
\end{aligned}
$$

The boundary condition is that the sum of the relative subpopulations of all species is 1 : 


$$
P_{\mathrm{E}}([\mathrm{S}], t)+P_{\mathrm{ES}}^{*}([\mathrm{~S}], t)+P_{\mathrm{ES}}([\mathrm{S}], t)=1
$$

When $t \rightarrow \infty$, the system reaches equilibrium. The subpopulations of $P_{\mathrm{E}}([\mathrm{S}], t), P_{\mathrm{ES}}([\mathrm{S}], t), P_{\mathrm{ES}}{ }^{*}([\mathrm{~S}], t)$ no longer change with $t$, and were simplified to $P_{\mathrm{E}}([\mathrm{S}]), P_{\mathrm{ES}}([\mathrm{S}]), P_{\mathrm{ES}}([\mathrm{S}])$. Eq S28 to S31 at $t \rightarrow \infty$ were rewritten as:

$$
\begin{gathered}
k_{-1} P_{\mathrm{ES}}{ }^{*}([\mathrm{~S}])+k_{-3} P_{\mathrm{ES}}([\mathrm{S}])-\left(k_{1}+k_{3}\right)[S] P_{\mathrm{E}}([\mathrm{S}])=0 \\
k_{3}[\mathrm{~S}] P_{\mathrm{E}}([\mathrm{S}])+k_{2} P_{\mathrm{ES}}{ }^{*}([\mathrm{~S}])-\left(k_{-3}+k_{-2}\right) P_{\mathrm{ES}}([\mathrm{S}])=0 \\
k_{1}[\mathrm{~S}] P_{\mathrm{E}}([\mathrm{S}])+k_{-2} P_{\mathrm{ES}}([\mathrm{S}])-\left(k_{-1}+k_{2}\right) P_{\mathrm{ES}}{ }^{*}([\mathrm{~S}])=0 \\
P_{\mathrm{E}}([\mathrm{S}])+P_{\mathrm{ES}}^{*}([\mathrm{~S}])+P_{\mathrm{ES}}([\mathrm{S}])=1
\end{gathered}
$$

By solving Eq S32 to Eq S35, the relative population of each species is expressed in term of rate constants and substrate concentration [S]:

$$
\begin{aligned}
P_{\mathrm{E}}([\mathrm{S}]) & =\frac{k_{2} k_{-3}+k_{-1}\left(k_{-2}+k_{-3}\right)}{k_{2}\left(k_{-3}+\left(k_{1}+k_{3}\right)[\mathrm{S}]\right)+k_{3} k_{-2}[\mathrm{~S}]+k_{1}\left(k_{-2}+k_{-3}\right)[\mathrm{S}]+k_{-1}\left(k_{-2}+k_{-3}+k_{3}[\mathrm{~S}]\right)} \\
P_{\mathrm{ES}}{ }^{*}([\mathrm{~S}]) & =\frac{\left(k_{3} k_{-2}+k_{1}\left(k_{-2}+k_{-3}\right)\right)[\mathrm{S}]}{k_{2}\left(k_{-3}+\left(k_{1}+k_{3}\right)[\mathrm{S}]\right)+k_{3} k_{-2}[S]+k_{1}\left(k_{-2}+k_{-3}\right)[\mathrm{S}]+k_{-1}\left(k_{-2}+k_{-3}+k_{3}[\mathrm{~S}]\right)} \\
P_{\mathrm{ES}}([\mathrm{S}]) & =\frac{\left(k_{1} k_{2}+k_{3}\left(k_{2}+k_{-1}\right)\right)[\mathrm{S}]}{k_{2}\left(k_{-3}+\left(k_{1}+k_{3}\right)[\mathrm{S}]\right)+k_{3} k_{-2}[\mathrm{~S}]+k_{1}\left(k_{-2}+k_{-3}\right)[\mathrm{S}]+k_{-1}\left(k_{-2}+k_{-3}+k_{3}[\mathrm{~S}]\right)}
\end{aligned}
$$

\section{Computation cost for SMIS simulation and fittings}

All simulations and fitting were performed on the commercially available desktop (CPU: Intel ${ }^{\circledR}$ Core $^{\mathrm{TM}}$ i7-8700 CPU @ 3.20GHz 3.19 GHz, RAM: 32.0 GB, OS: Win 1064 bit). For the five reaction species associating with a three FRET level system (total eight rate constants), the time to generate one set of simulated dwell-time distributions, average dwell time, and relative populations is around 1s. Regarding the extraction of reaction rate constants using SMIS, the computation time will depend on the grid size of choice. Using a grid size of 5 different values for each rate constant and the number of transition set to 100,000 , it will take $\sim 7 \mathrm{hr}$ to obtain the $\%$ RSD and corresponding rate-constant distribution. For a fourround optimization search, it will take $\sim 28 \mathrm{hr}$.

\section{SMIS validation for Michaelis-Menten model (other four conditions)}

In this section, we validated the SMIS by comparing the simulation results with the analytical solutions of the Michaelis-Menten model. Figure S3, Figure S4, and Figure S5 respectively shows the comparison of the dwell-time distribution (Section 3.1), the average transition rates (Section 3.2), and relative populations of each species (Section 3.3) between the analytical and simulated results. In all conditions, the simulations (blue bar or circle) nicely overlap with the prediction (red line), suggesting the simulation successfully reproduced the expected results. 


\subsection{Validation of simulated $f_{\mathrm{HL}}(\tau)$ and $f_{\mathrm{LH}}(\tau)$ as a function of $k$}

a

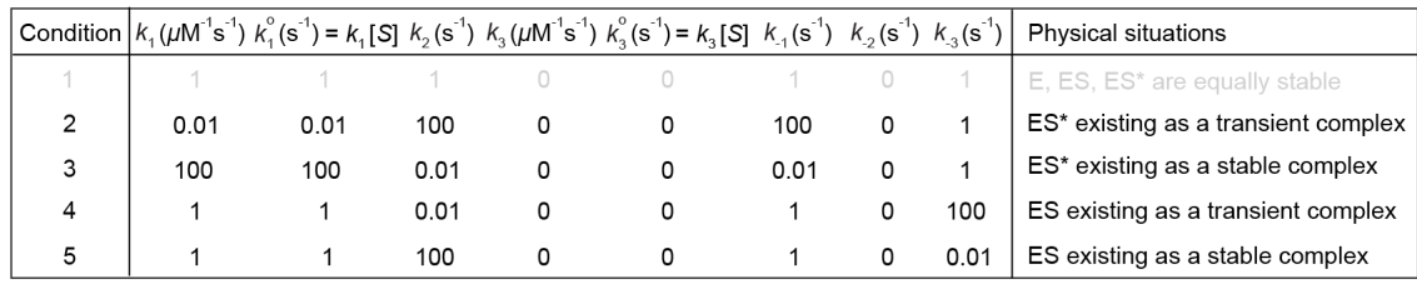

b Condition 2

C Condition 3

d Condition 4

Condition 5
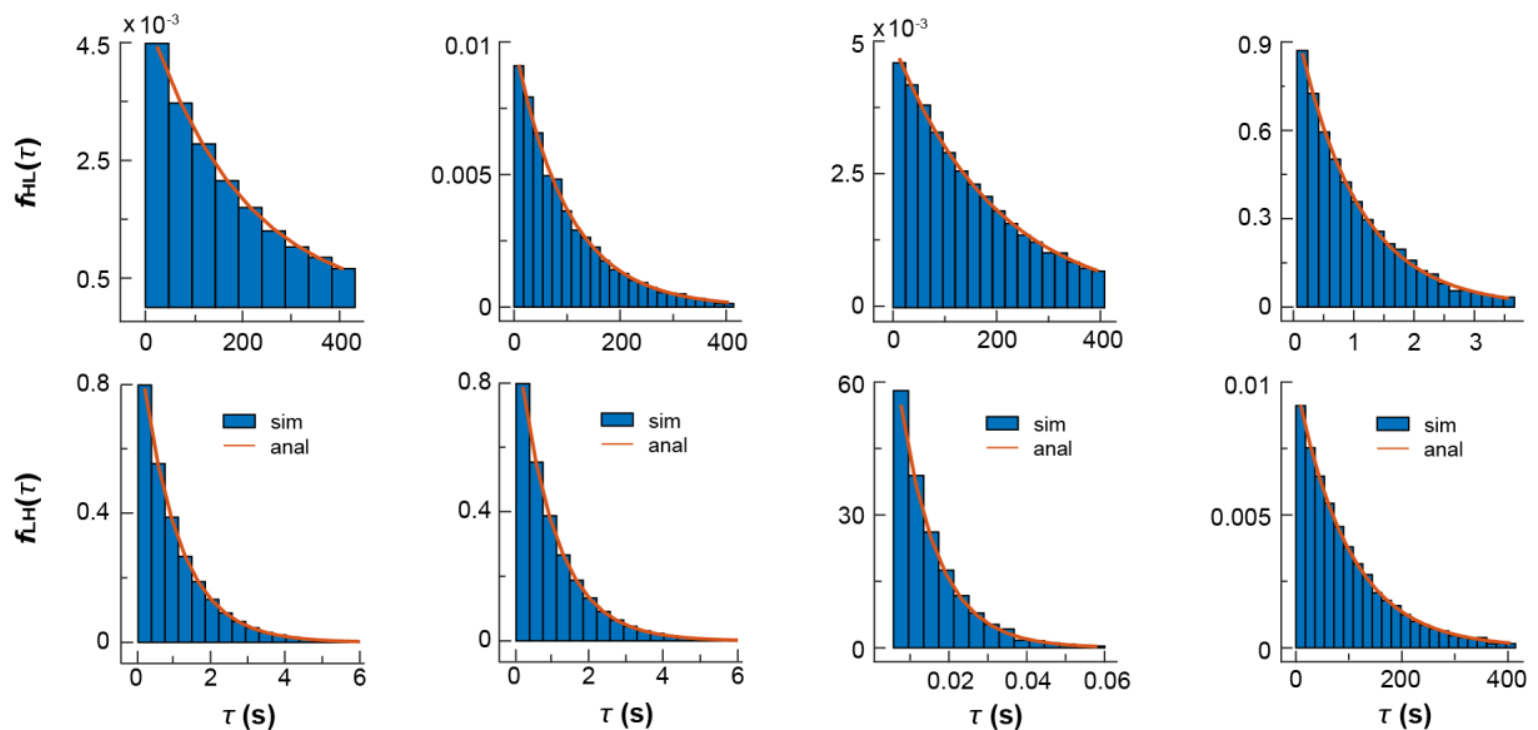

Figure S3. Comparisons between simulated (blue bar) and analytical (red line) $f(\tau)$ for the Michaelis-Menten enzyme kinetic model under condition 2-5. (a) Rate constants for the SMIS simulation and analytical solution of the Michaelis-Menten model. (b-e) Simulation of the probability density function $f_{\mathrm{HL}}(\tau)$ and $f_{\mathrm{LH}}(\tau)$ under condition $2(\mathbf{b})$, condition 3 (c), condition 4 (d) and condition 5 (e).

\subsection{Validation of simulated $\left\langle\tau_{\mathrm{HL}}\right\rangle^{-1}$ and $\left\langle\tau_{\mathrm{LH}}\right\rangle^{-1}$ as a function of $k$}

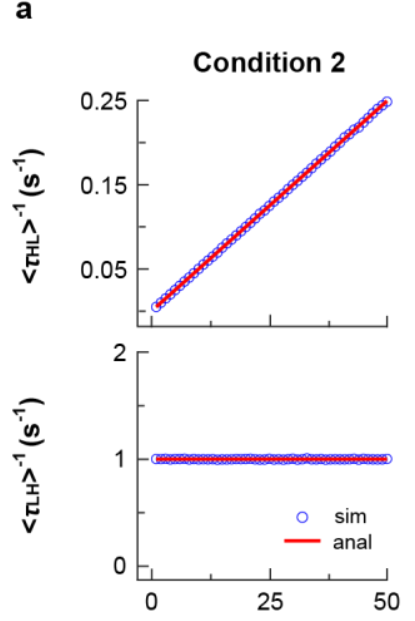

$[\mathrm{S}](\mu \mathrm{M})$

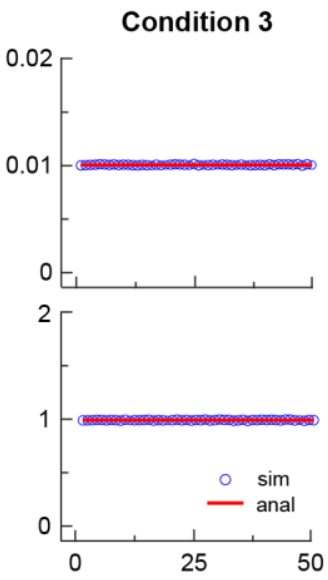

$[\mathrm{S}](\mu \mathrm{M})$

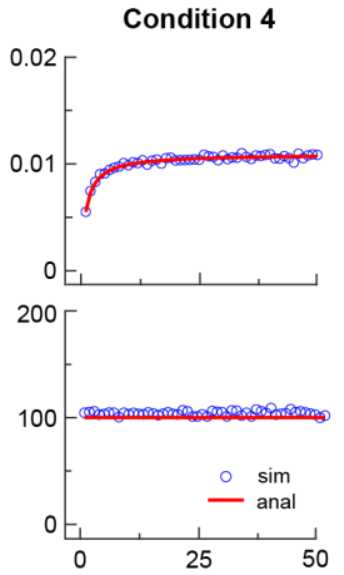

$[\mathrm{S}](\mu \mathrm{M})$ d

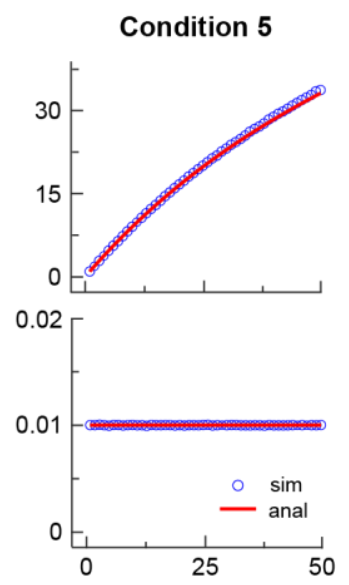

[S] ( $\mu \mathrm{M})$

Figure S4. Comparisons between simulated (blue circle) and analytical (red line) $\langle\tau\rangle^{-1}$ for the Michaelis-Menten enzyme kinetic model under condition 2-5. (a-d) Simulation of the average transition rate $\left\langle\tau_{\mathrm{HL}}\right\rangle^{-1}$ and $\left\langle\tau_{\mathrm{LH}}\right\rangle^{-1}$ under condition $2(\mathbf{a})$, condition 3 (b), condition 4 (c) and condition 5 (d). 


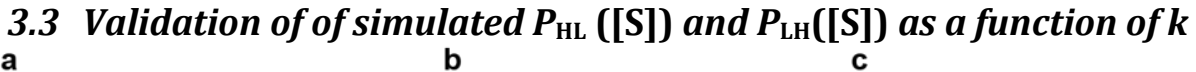

a

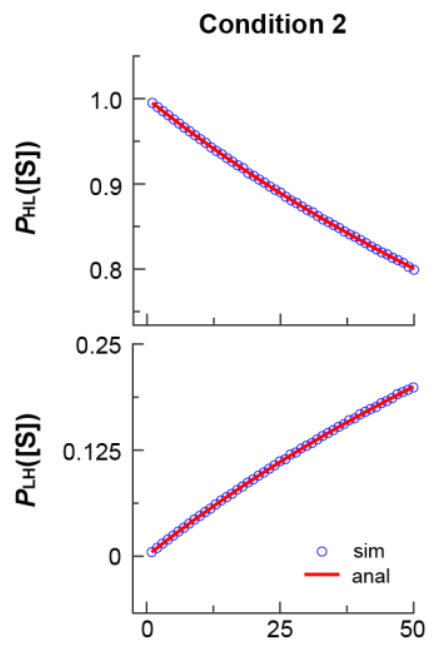

[S] $(\mu \mathrm{M})$

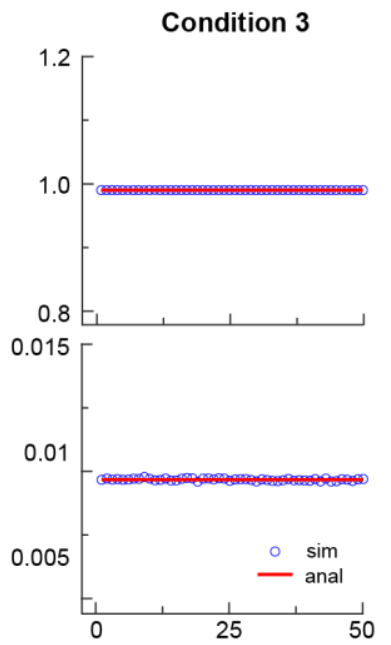

[S] $(\mu \mathrm{M})$

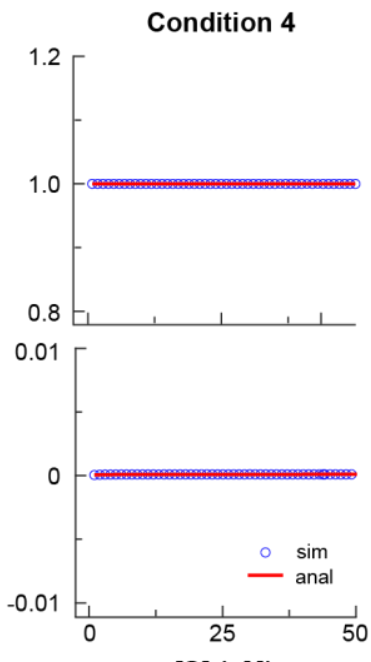

d

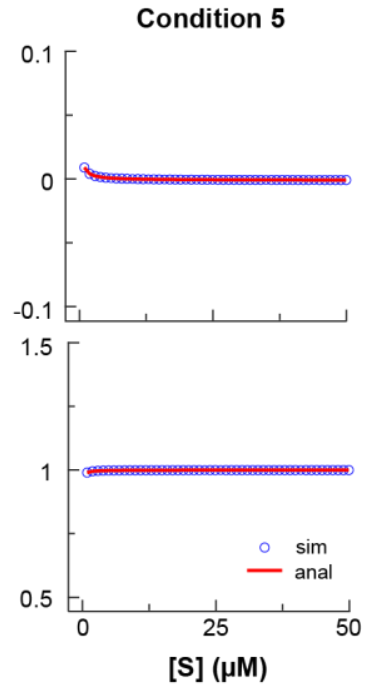

Figure S5. Comparisons between simulated (blue circle) and analytical (red line) populations for the Michaelis-Menten enzyme kinetic model under condition 2-5. (a-d) Simulation of the relative populations $P_{\mathrm{HL}}$ and $P_{\mathrm{LH}}$ under condition 2 (a), condition 3 (b), condition 4 (c) and condition 5 (d).

\section{SMIS validation using a kinetic model having five reaction species in 3 FRET levels}

We extended the application of SMIS to the kinetic model with two intermediates in three FRET states (Figure S6a) whose analytical solution is difficult to obtain. The kinetic model describes an enzyme existing as one of the five interacting species $\left(\mathrm{E}, \mathrm{ES}^{*}, \mathrm{ES}, \mathrm{ES}_{2}{ }^{*}\right.$, and $\left.\mathrm{ES}_{2}\right)$ where $\mathrm{E}$ and $\mathrm{ES}^{*}$ associates with the FRET high $\left(I_{\mathrm{H}}\right)$ state; ES and $\mathrm{ES}_{2} *$ with the middle $\left(I_{\mathrm{M}}\right)$ state; and $\mathrm{ES}_{2}$ with the low $\left(I_{\mathrm{L}}\right)$ state. The substrate $\mathrm{S}$ binds to the enzyme $\mathrm{E}$ to form the interacting complex ES through different intermediates ES* and $\mathrm{ES}_{2} *$ with the forward $\left(k_{1}, k_{2}, k_{3}\right.$, and $\left.k_{4}\right)$ and reversed $\left(k_{-1}, k_{-3}, k_{-5}\right.$, and $\left.k_{-6}\right)$ rate constants annotated. We randomly selected a set of rate constants as specified in Figure $\mathbf{S 6 \mathbf { b }}$. The simulated $P D F_{\mathrm{HL}}(\tau), P D F_{\mathrm{LH}}(\tau)$, $P_{\mathrm{HL}}$, and $P_{\mathrm{LH}}$ under three substrate concentrations $([\mathrm{S}]=5,20$, and $60 \mu \mathrm{M})$, as shown in Figure S6c. To search for the most probable rate constants, we applied the repeated bisection method to each rate-constant histogram. Figure S6d shows the results for each rate constant after two searches. With this approach, we extracted all rate constants that are in agreement with the inputs. 
a

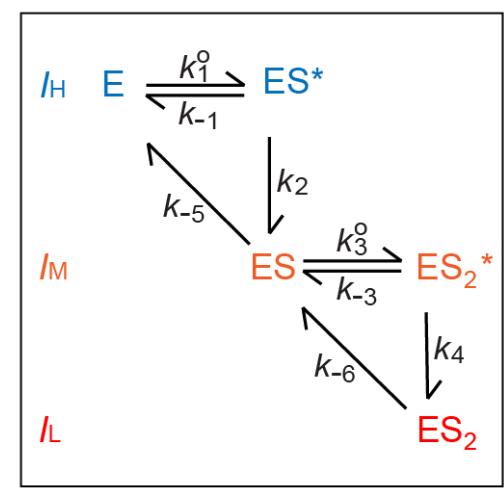

Note: $k_{1}^{\circ}=k_{1}[S], k_{3}^{\circ}=k_{3}[S]$
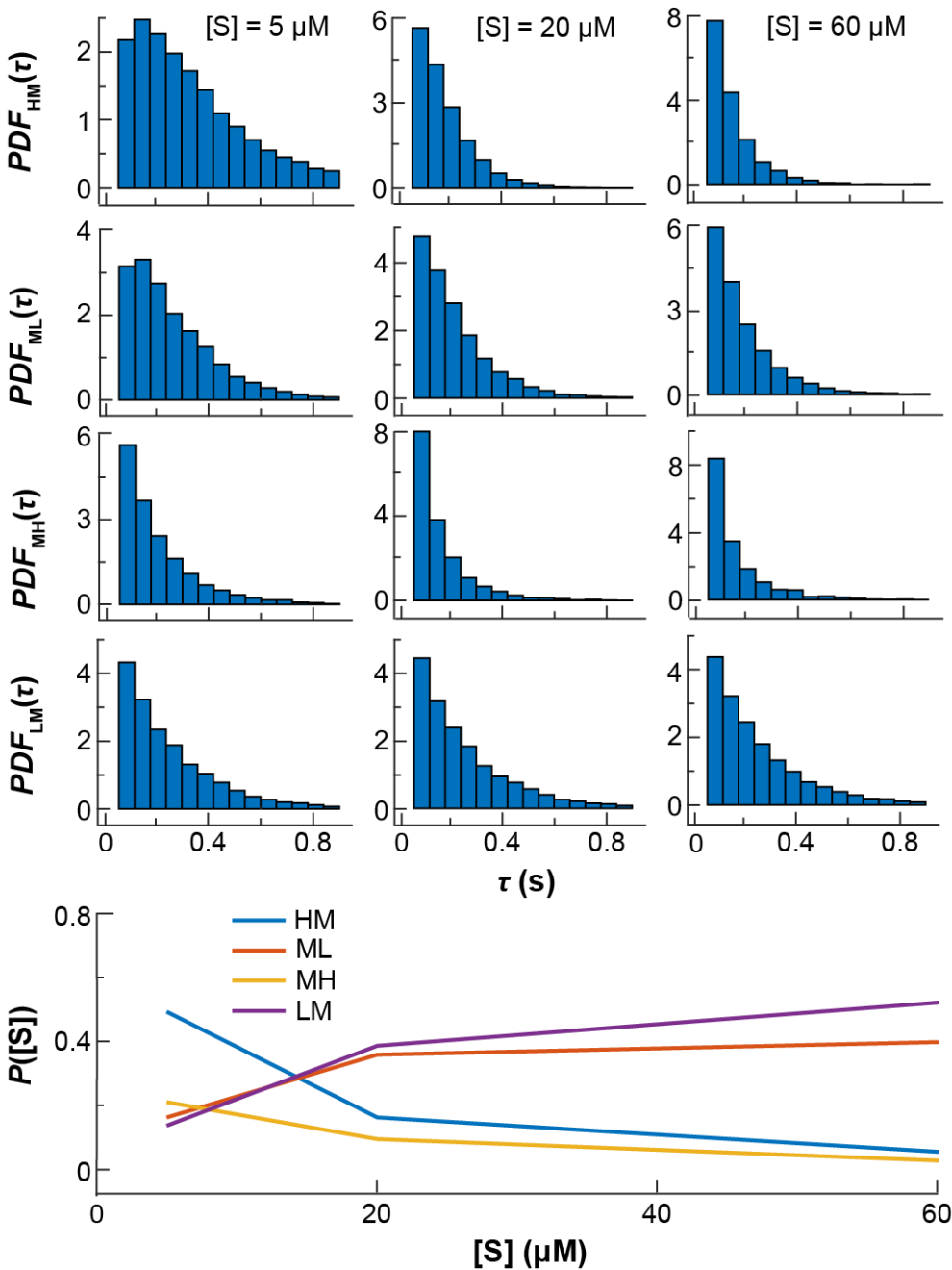

b

\begin{tabular}{ccccc}
\hline \multirow{2}{*}{ Kinetic paramaters } & \multirow{2}{*}{ Input value } & \multicolumn{3}{c}{ Extracted value } \\
\cline { 3 - 5 } & & MPV & LB & UB \\
\hline$k_{1}\left(\mu \mathrm{M}^{-1} \mathrm{~s}^{-1}\right)$ & 0.8 & 0.7 & 0.0 & 8.1 \\
$k_{2}\left(\mathrm{~s}^{-1}\right)$ & 11 & 6.1 & 0.6 & 11.6 \\
$k_{3}\left(\mu \mathrm{M}^{-1} \mathrm{~s}^{-1}\right)$ & 0.6 & 0.6 & 0.0 & 4.6 \\
$k_{4}\left(\mathrm{~s}^{-1}\right)$ & 8 & 6.2 & 2.6 & 9.9 \\
$k_{-1}\left(\mathrm{~s}^{-1}\right)$ & 0.2 & 0.0 & 0.0 & 9.4 \\
$k_{-3}\left(\mathrm{~s}^{-1}\right)$ & 2.5 & 5.9 & 1.0 & 10.7 \\
$k_{-5}\left(\mathrm{~s}^{-1}\right)$ & 5 & 6.0 & 0.9 & 11.1 \\
$k_{-6}\left(\mathrm{~s}^{-1}\right)$ & 5 & 6.0 & 3.8 & 8.1 \\
\hline
\end{tabular}

Note: MPV: most probable value; LB: lower bound; UB: upper bound

d
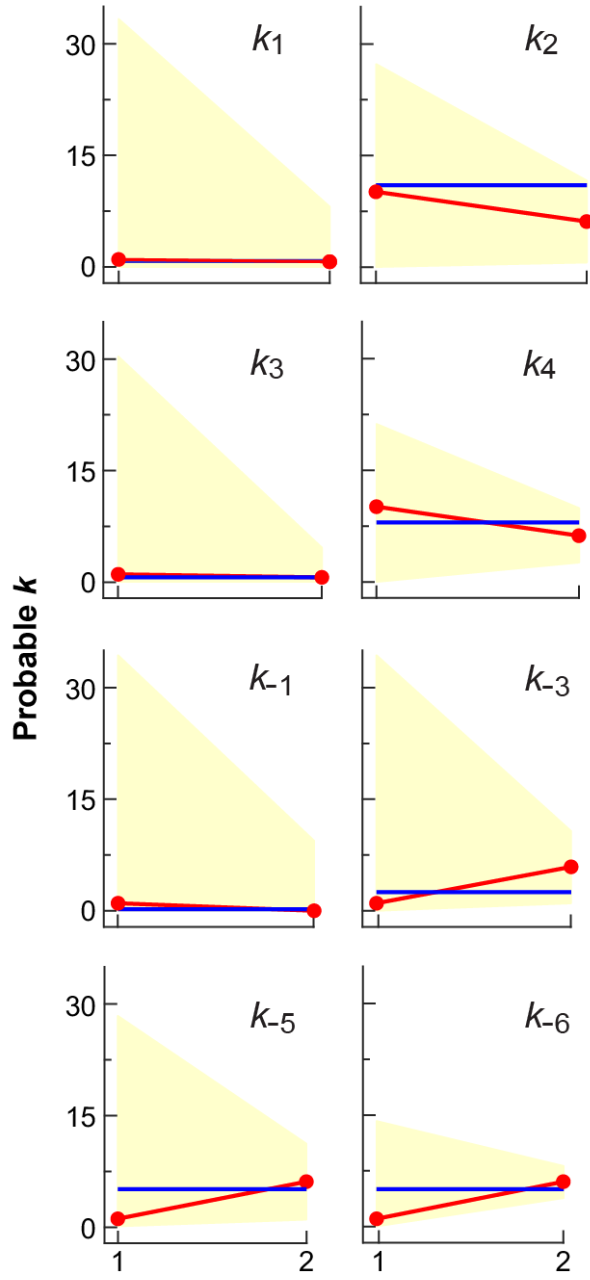

Simulation index

Figure S6. SMIS validation using a kinetic model with five reaction species in 3 FRET levels. (a) The kinetic model describes five interacting species (E, ES*, ES, $\mathrm{ES}_{2}{ }^{*}$, and $\mathrm{ES}_{2}$ ) associated with three FRET levels $\left(I_{\mathrm{H}}, I_{\mathrm{M}}\right.$, and $\left.I_{\mathrm{L}}\right)$. (b) Summary of input and extracted rate constants obtained from SMIS. (c) SMIS simulation results of $P D F_{\mathrm{HM}}(\tau), P D F_{\mathrm{ML}}(\tau), P D F_{\mathrm{MH}}(\tau), P D F_{\mathrm{LM}}(\tau)$, and $[\mathrm{S}]$ dependent population $\left(P_{\mathrm{HM}}, P_{\mathrm{ML}}, P_{\mathrm{MH}}\right.$ and $\left.P_{\mathrm{LM}}\right)$ under 3 substrate concentrations $([\mathrm{S}]=5$, 20, and $60 \mu \mathrm{M})$. (d) Progression of identifying correct rate constants. The yellow area highlights the screening range of $k$ in each screening. Bluelines indicate the input rate constants for the experimental data. Redlines show the most probable rate constant extracted from SMIS. 